Journal of Computer Science 6 (8): 896-903, 2010

ISSN 1549-3636

(C) 2010 Science Publications

\title{
Development of an Ultra-Miniaturized Inertial Measurement Unit for Jaw Movement Analysis during Free Chewing
}

\author{
Zhuohua Lin, Massimiliano Zecca, Salvatore Sessa, Hiroyuki Ishii and Atsuo Takanishi \\ Department of Modern Mechanical Engineering, \\ School of Advanced Science and Engineering, Waseda University, Tokyo 168-8480, Japan
}

\begin{abstract}
Problem statement: Jaw movement analysis, as a clinical aid, can provide an objective basis for understanding and diagnosing jaw musculoskeletal disorders. Therefore, the use and development of devices for quantitatively measuring and analyzing jaw movement have become more common and popular in the clinic. Many types of jaw tracking devices have been developed, but most of them are still not handy and easy to be used. Approach: To improve the handiness and utility of the jaw movement analysis devices, we developed a simple to be used jaw tracking prototype by using a new ultra-miniaturized Inertial Measurement Unit (IMU) named WB-3. The WB-3 IMU was composed by 3-axis gyroscope, 3-axis accelerometer and 3-aixs magnetometer, which can not only measure the acceleration and angular speed of jaw movement, but also can measure mouth opening angle. The IMU's extremely reduced weight and size allowed it to be easily adhered to mandible during normal tests without physical restriction to the subjects. A preliminary experiment for jaw movement analysis during free chewing of three types of food with different shapes and hardness was evaluated. A group of 15 healthy subjects aged from 21-36 years old kindly participated in the experiment. Results: The parameters of chewing time, chewing frequency, power spectrum density of jaw's angular speed and acceleration, cumulative distribution function of jaw's acceleration and mouth opening angle were presented. The experimental results clearly showed that the subjects used less chewing time, less chewing frequency, less acceleration cumulative distribution and energy to eat soft food; higher values were found in the case of hard food and there was no significant difference in mouth opening angle while eating these three foods. Conclusion: Our jaw movement analysis prototype using IMU WB-3 was proved to be a valid and handy method for jaw movement and pattern analysis which may be used clinically as an assistant system for dental therapy.
\end{abstract}

Key words: Inertial measurement unit, jaw tracking, mastication analysis

\section{INTRODUCTION}

The analysis of jaw movement has long been used as a measure for clinical diagnosis and treatment of prosthodontics, orthodontics and oral surgery. Such as the Temporomandibular Joint and Muscle Disorders (TMJDs), between 5 and $15 \%$ of people in the United States experience pain associated with this disease (Kuska, 2005). The goal of jaw movement analysis for this disease has been to provide an objective basis for clinical diagnosis or to monitor the progress of active treatment methods. The extent to which jaw tracking provides a useful research tool, a diagnostic aid, or a therapeutic monitor clearly depends on what is being measured, how the process is carried out and why the information is important (Soboleva et al., 2005; Otake et al., 2006).
Therefore, the use and development of devices for quantitatively measuring and analyzing jaw movement has recently become more common and popular in the clinic (Naeije and Hofman, 2003).

Conventional mechanical methods, such as JMA system of Zebris Company, usually use special mechanisms put on the head in order to capture jaw movement. These mechanisms are a little bulky and make the patients feel not natural during the routine tests. Conventional optical methods for jaw movement analysis usually have adverse effect due to the presence of metal clutches in the patient's mouth. These clutches disturb the physiology and influence the jaw movement. Conventional magnetic methods have utilized a permanent magnet and magnetic field sensors, but these methods have the inherent limitation that the

Corresponding Author: Massimiliano Zecca, Department of Modern Mechanical Engineering,

School of Advanced Science and Engineering, Waseda University, Tokyo 168-8480, Japan

Tel: 81-03-5369-7369/Ext: 3604 Fax: 81-03-5269-9061 
system provides only the position of the point source and then obtain rotations and accelerations by successive derivations which introduce more noise (Yabukami et al., 2002). In most cases, the systems that have been described are relatively bulky, heavy or not handy, complicated to be used and expensive (Flavel et al., 2002; Kitamura, 2006).

Our aim, therefore, is to develop evaluation tools and methodologies to overcome the limitations of the existing devices and improve the affectivity and handiness of tools for jaw movement analysis. In this study, we present a simple to be used jaw tracking prototype which uses a new ultra-miniaturized and inexpensive Inertial Measurement Unit (IMU) named WB-3 for jaw movement analysis. The IMU's extremely reduced weight and size allows it to be easily adhered to the mandible during normal tests without physical restriction to the subjects. A preliminary experiment for jaw movement analysis during free chewing of three types of food with different shapes and hardness was elaborated to evaluate the system validity.

This study is organized as follows. "Materials and Methods" part presents the WB-3 Inertial Measurement Unit, the experimental setup and the experimental protocol. The experimental analysis of the results is presented in the "Results". Discussion and Conclusion follow.

\section{MATERIALS AND METHODS}

WB-3 Inertial Measurement Unit (IMU): Our group recently developed a new IMU named WB-3, which is very compact and lightweight (size $26 \times 20 \times 8 \mathrm{~mm}$ and weight $2.9 \mathrm{~g}$ )-at present the smallest and, lightest in the world at the time of its development (Zecca et al., 2009; Sessa et al., 2009). A picture of the new IMU is shown in Fig. 1. The IMU is composed by the following sensors: 3-axis accelerometer LIS3LV02DL; 2-axis gyroscope IDG300; 1-axis gyroscope LISY300AL and 3-axis magnetometer HMC5843. The IMU's characteristics have been summarized in Table 1 .

All the sensors have 12 bit resolution. The LIS3LV02DL (STMicroelectronics) is a 3 -axis accelerometer, whose small size $(4.4 \times 7.5 \times 1 \mathrm{~mm})$ and high performance characteristics are fully compatible with the requirements of jaw movement tracking. The sensitivity with a full-scale $= \pm 2 \mathrm{~g}\left(1 \mathrm{~g}=9.8 \mathrm{~m} \mathrm{sec}^{-2}\right)$ and bandwidth $=40 \mathrm{~Hz}$ is about $0.001 \mathrm{~g}$, with noise level less than one bit. The LISY300AL (STMicroelectronics) is a miniaturized $7.0 \times 7.0 \times 1.9 \mathrm{~mm}$ z-axis gyro sensor. Its full-scale is $\pm 300 \mathrm{deg} \mathrm{sec}{ }^{-1}$ with a bandwidth of $88 \mathrm{~Hz}$ and a sensibility of $3.3 \mathrm{mV} \mathrm{deg}-1$ $\mathrm{sec}^{-1}$. In order to measure 3 -axes angular velocities, we also used a bi-axial gyro IDG300 (InvenSense). The IDG300 size is $6.0 \times 6.0 \times 1.5 \mathrm{~mm}$, the measurement range is $\pm 500 \mathrm{deg} \mathrm{sec}^{-1}$ and the

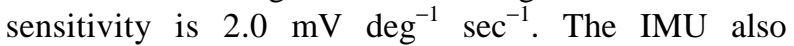
contains a 3-axis magnetometer HMC5843 whose size is $4.0 \times 4.0 \times 1.5 \mathrm{~mm}$ and full scale is \pm 4 Gauss with a noise level less than two bits. Unlike all other prototypes and commercial IMUs available today, this mixed configuration allows our IMU to obtain all the 3 axis of the gyros in one planar layer.

Our IMU also contains a STMicroelectronics 32 bit microcontroller STM32F103CB for embedded signal elaboration and data exchange. The communication between PC and IMU is performed by using CAN BUS at $1 \mathrm{Mb} \mathrm{sec}^{-1}$. Data are acquired at $100 \mathrm{~Hz}$ and stored on a personal computer for post-processing.

Experimental setup: The experimental setup is shown in Fig. 2. One WB-3 IMU was adhered to subject's mandible in order to measure the jaw movement while freely chewing three different foods: marshmallow, biscuit and almond, shown in Fig. 3, whose weights were $8.0 \pm 1.0,7.0 \pm 0.1$ and $4.3 \pm 0.4 \mathrm{~g}$, respectively. The experimental foods were chosen from the everyday living to represent different hardness of foods. The subject was instructed to lean his head on the wall during chewing the food in order to minimize the influence of head's movement.

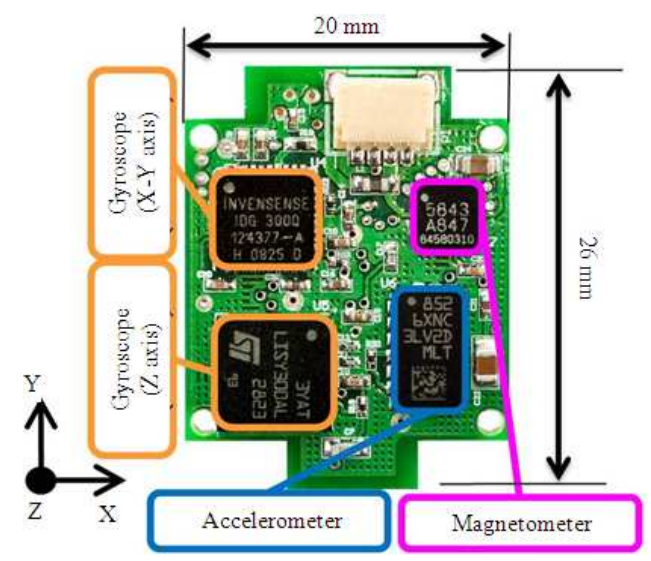

Fig. 1: Inertial measurement unit WB-3

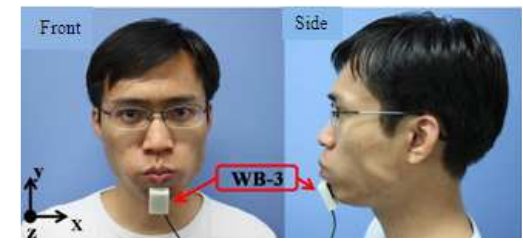

Fig. 2: Experimental setup 
Table 1: Main characteristics of the sensors in WB-3

\begin{tabular}{lllll}
\hline & LIS3LV02DL & IDG300 & LISY300AL & HMC5843 \\
\hline Category & Accelerometer & Gyro & Gyro & Magnetometer \\
Axis & 3 -axis & 2 -axis & 1 -axis & 3 -axis \\
Range & $\pm 2(\mathrm{~g})$ & $\pm 500\left(\mathrm{deg} \mathrm{sec}^{-1}\right)$ & $\pm 300\left(\mathrm{deg} \mathrm{sec}^{-1}\right)$ & $\pm 4\left(\mathrm{gauss}^{-1}\right)$ \\
Resolution & $12 \mathrm{bits}$ & $12 \mathrm{bits}$ & $12 \mathrm{bits}$ & $12 \mathrm{bits}$ \\
Bandwidth & $40 \mathrm{~Hz}$ & $140 \mathrm{~Hz}$ & $88 \mathrm{~Hz}$ & $40 \mathrm{~Hz}$ \\
Linearity & $\pm 2 \%$ & $<1 \%$ & $\pm 0.8 \%$ & $\pm 0.1 \%$ \\
Noise level & $<1 \mathrm{bit}$ & $<2 \mathrm{bit}$ & $<2 \mathrm{bit}$ & $<2 \mathrm{bit}$ \\
\hline
\end{tabular}

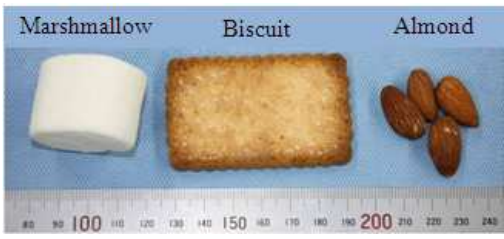

Fig. 3: Three experimental foods

Experimental protocol: Thirteen male and two female subjects (age range of 21-36 years) with healthy masticator systems and complete dentition participated in the study after providing informed consent. Each subject ate the food by following the order:

$$
\text { Marshmallow } \rightarrow \text { biscuit } \rightarrow \text { almond }
$$

Each food was tested 3 times. Therefore, 9 trials were organized for each subject ( 3 foods $\times 3$ times). At the beginning of each trial, subject put the food inside the mouth and kept mouth closed. A trial was defined to start chewing the food and stop at swallowing down the food. Subjects were instructed to chewing the foods freely as usual as their regular eating habits.

All the data were sampled at frequency $\mathrm{f}=100 \mathrm{~Hz}$ and saved as Comma Separated Value (CSV) and then loaded in MATLAB ${ }^{\circledR}$ (The Math Works, Inc.) for further processing and analysis. Acceleration components, angular speed components and magnetic components were filtered and smoothed by using a 10th order bandpass IIR Butterworth filter with cutoff frequencies $\mathrm{f}_{\mathrm{c} 1}=0.05 \mathrm{~Hz}, \mathrm{f}_{\mathrm{c} 2}=5 \mathrm{~Hz}$, to remove bias and physiological tremor (Ibanez et al., 2006; Veluvolu et al., 2007). In this way only the data due to the voluntary jaw movement were analyzed.

\section{RESULTS}

The details about the experimental evaluation were presented. In particular, the following variables were calculated and analyzed: Chewing Time (CT); Chewing Frequency (CF); Power Spectrum Density (PSD) of jaw's $\mathrm{x}$-axis angular speed $\omega_{\mathrm{x}}$ and acceleration module $|\mathrm{a}|$; Cumulative Distribution Function (CDF) of jaw's acceleration module $|\mathrm{a}|$ and Mouth Opening Angle (MOA).

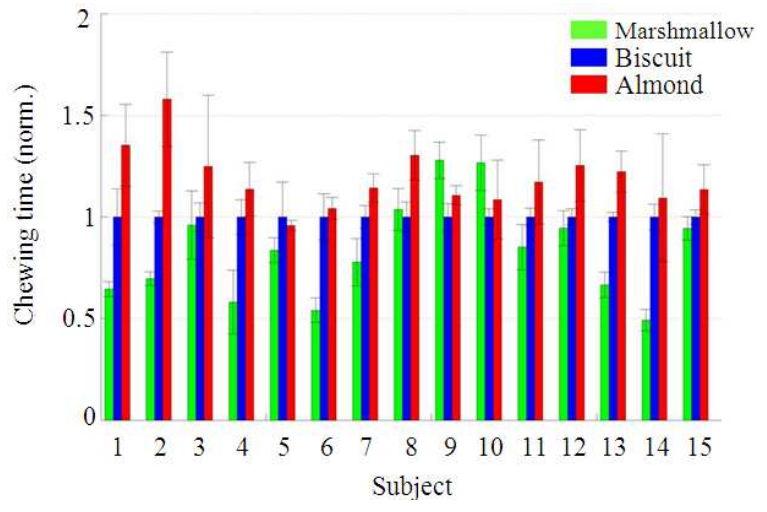

Fig. 4: Normalized chewing time for 3 different foods, averaged on the 3 trials for each subject

These parameters are related to the masticator efficiency and pattern while chewing different foods. The Fast Fourier Transformation (FFT) for acceleration and angular speed was calculated with $\mathrm{FFT}_{\text {size }}=8192$ samples and frequency resolution fres $=$ f/FFTsize $=$ $100 \mathrm{~Hz} / 8192=0.0122 \mathrm{~Hz}$. The PSD was estimated in using the following formula:

$$
\text { (FFT· conj (FFT))/FFT }{ }_{\text {size }}
$$

The frequency range chosen for the evaluation was $0.1-5 \mathrm{~Hz}$ to take into account only the voluntary movements. The CDF of acceleration $|\mathrm{a}|$ was calculated as $\operatorname{CDF}(|\mathrm{a}|)=\mathrm{P}(\mathrm{X} \leq|\mathrm{a}|)$ evaluated for $\mathrm{X}=95 \%$ $\left(\mathrm{CDF}_{95 \%}\right)$. The orientation estimate of IMU for calculating the mouth opening angle is based on the Extended Kalman Filter algorithm by fusing the data from gyroscope, accelerometer and magnetometer (Marins et al., 2001). From Fig. 4-16, (norm.) indicates that each subject's data have been normalized to his average value of biscuit in order to remove the effect of inter-subject anatomical difference. The normalization values are summarized in Table 2.

Chewing time: Most subjects proved to be usually using less time in chewing marshmallow than chewing almond, as shown in Fig. 4. Only subject 9 and 10 happened to be using more time for marshmallow than almond. 
Table 2: Normalization values of each subject

\begin{tabular}{|c|c|c|c|c|c|c|}
\hline Subject & $\mathrm{CT}(\mathrm{sec})$ & $\mathrm{CF}(\mathrm{Hz})$ & $\left.P S D \omega_{x}\left[(\operatorname{deg~sec})^{-1}\right)^{2}\right]$ & PSD $|\mathrm{a}|\left[\left(\mathrm{m} \mathrm{sec}^{-2}\right)^{2}\right]$ & $\mathrm{CDF}|\mathrm{a}|_{95 \%}\left(\mathrm{~m} \mathrm{sec}^{-2}\right)$ & MOA (deg) \\
\hline 1 & 31.2 & 1.57 & $8.0 \mathrm{e}+03$ & 3.15 & 1.70 & 17.1 \\
\hline 2 & 43.9 & 1.35 & $1.1 \mathrm{e}+04$ & 2.67 & 1.22 & 18.5 \\
\hline 3 & 32.6 & 1.66 & $3.5 e+03$ & 7.59 & 1.89 & 11.0 \\
\hline 4 & 19.5 & 1.72 & $4.2 \mathrm{e}+03$ & 4.75 & 1.42 & 13.2 \\
\hline 5 & 27.0 & 1.46 & $3.0 \mathrm{e}+03$ & 4.62 & 1.34 & 8.5 \\
\hline 6 & 21.6 & 1.89 & $5.5 \mathrm{e}+03$ & 4.00 & 1.30 & 12.8 \\
\hline 7 & 37.4 & 1.62 & $6.7 \mathrm{e}+03$ & 4.43 & 1.38 & 10.8 \\
\hline 8 & 22.7 & 1.55 & $3.2 \mathrm{e}+03$ & 4.68 & 1.34 & 11.6 \\
\hline 9 & 24.7 & 1.72 & $8.5 e+03$ & 5.34 & 1.48 & 15.9 \\
\hline 10 & 42.0 & 1.22 & $3.5 \mathrm{e}+03$ & 1.87 & 0.92 & 10.6 \\
\hline 11 & 17.5 & 1.68 & $1.1 \mathrm{e}+03$ & 3.64 & 1.21 & 7.5 \\
\hline 12 & 20.1 & 1.75 & $4.1 \mathrm{e}+03$ & 3.89 & 1.29 & 12.5 \\
\hline 13 & 33.1 & 1.72 & $3.7 \mathrm{e}+03$ & 5.51 & 1.47 & 11.9 \\
\hline 14 & 45.8 & 1.44 & $1.1 \mathrm{e}+04$ & 4.35 & 1.40 & 16.9 \\
\hline 15 & 33.5 & 1.40 & $3.8 \mathrm{e}+03$ & 3.70 & 1.23 & 8.5 \\
\hline
\end{tabular}

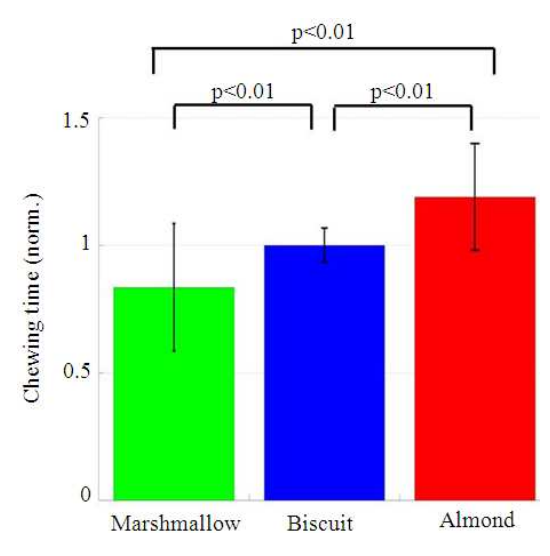

Fig. 5: Normalized chewing time for 3 different foods, averaged on all subjects' trials for each food

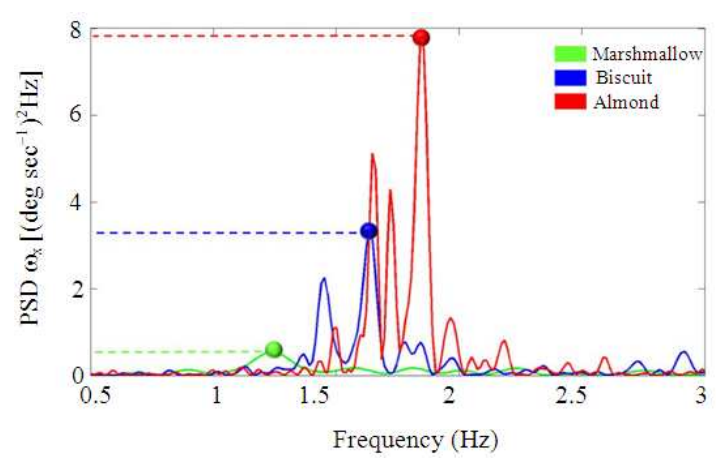

Fig. 6: Power Spectrum Density (PSD) of the $\mathrm{x}$-axis angular speed $\omega_{\mathrm{x}}$ for the subject 1 in trial 1

They were still using significantly more time for almond than for biscuit, though. As shown in Fig. 5, the chewing time for soft food averaged on all subjects' trials is significantly $(\mathrm{p}<0.01)$ lower than in case of the hard food. It is apparent that eating soft food is easier than hard food.

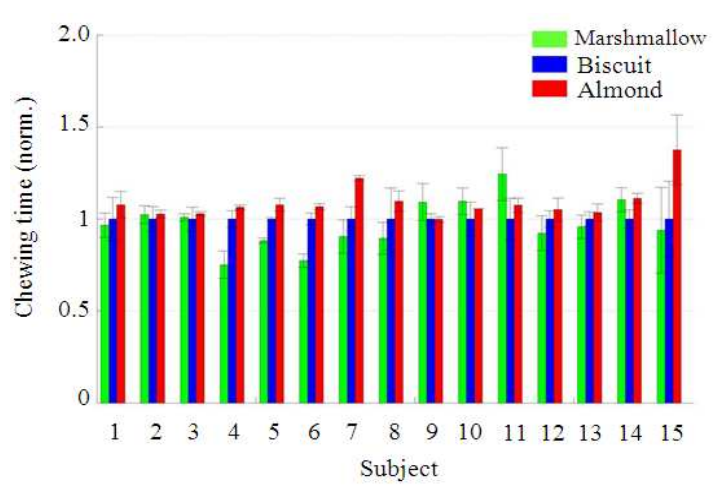

Fig. 7: Normalized chewing frequency for 3 different foods, averaged on the 3 trials for each subject

Chewing frequency: The chewing frequency was analyzed from the PSD of jaw's angular speed about xaxis (i.e., on the sagittal plane) whose rotation was more salient than $\mathrm{y}$ and z-axis's rotation during mastication. In Fig. 6, only subject 1's PSD of jaw's angular speed about $\mathrm{x}$-axis in one trial is shown, but similar results appear in case of the other subjects as well. The curves of all the foods had one maximum peak which is related to the highest PSD value. It means that the subject usually had one primary cycle for mastication which cost most energy. We used the frequency of this peak as the chewing frequency for each food.

As we can see in Fig. 7, most subjects were using higher chewing frequency when eating almonds. Only subject 9,10 and 11 happened to be using higher frequency for marshmallow. Subject 2 and 3 had almost equivalent values in eating all the three foods.

Fig. 8 clearly shows that subjects used significantly higher frequencies in eating almond than marshmallow or biscuits $(\mathrm{p}<0.01)$. This may indicate that the subjects used more energy in higher frequency for chewing the 
food in order to effectively chop and swallow down the food. There is no significant difference between marshmallows and biscuits.

Power Spectrum Density (PSD) of angular speed: The averaged PSD of jaw's angular speed about X-axis (on the sagittal plane) for all subjects is shown in Fig. 9. Most subjects used more rotation energy when eating hard food than soft food. Only subject 10 happened to use more energy for marshmallow. Figure 10 shows that subjects used significantly $(\mathrm{p}<0.01)$ more rotation energy when they were eating hard food.

Power Spectrum Density (PSD) of acceleration |a|: The averaged PSD of jaw's acceleration module $|\mathrm{a}|$ is shown in Fig. 11. Most subjects used more translation energy for hard food excluding subject 5 who used more translation energy for marshmallow. Figure 12 clearly shows that there was significant difference $(p<0.01)$ in PSD of acceleration while eating these three foods of different hardness. The hard food (almond) costs most translation energy for mastication.

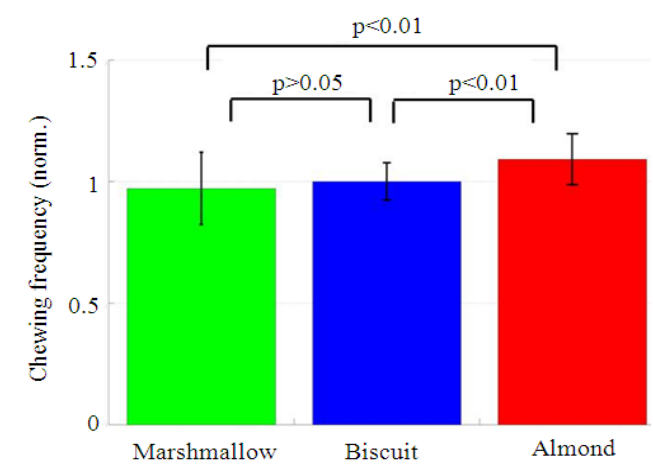

Fig. 8: Normalized chewing frequency for 3 different foods, averaged on all subjects' trials for each food

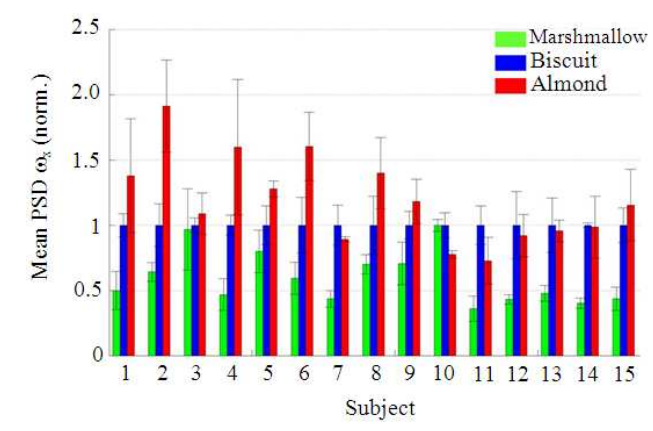

Fig. 9: Normalized Power Spectrum Density (PSD) of the $\mathrm{x}$-axis angular speed $\omega_{\mathrm{x}}$ for 3 different foods, averaged on the 3 trials for each subject
Cumulative Distribution Function (CDF) of acceleration |a|: The CDF of jaw's acceleration module $|\mathrm{a}|$ is shown in Fig. 13. Most subjects used higher $\mathrm{CDF}_{95 \%}$ for hard food, which may indicate that subjects used bigger force in chewing hard food.

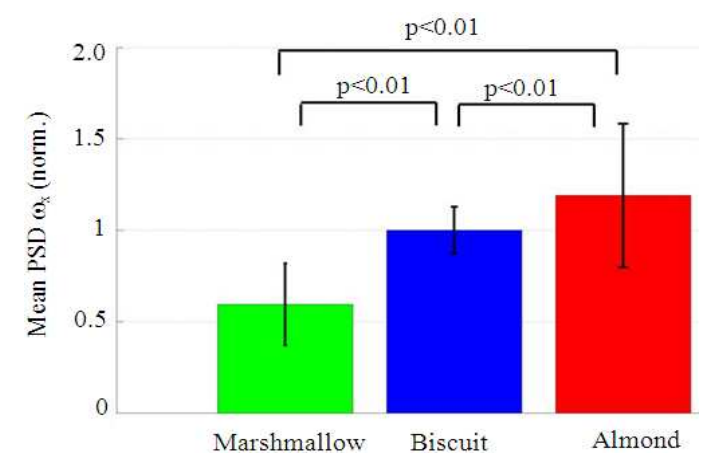

Fig. 10: Normalized Power Spectrum Density (PSD) of the $\mathrm{x}$-axis angular speed $\omega_{\mathrm{x}}$ for 3 different foods, averaged on all subjects' trials for each food

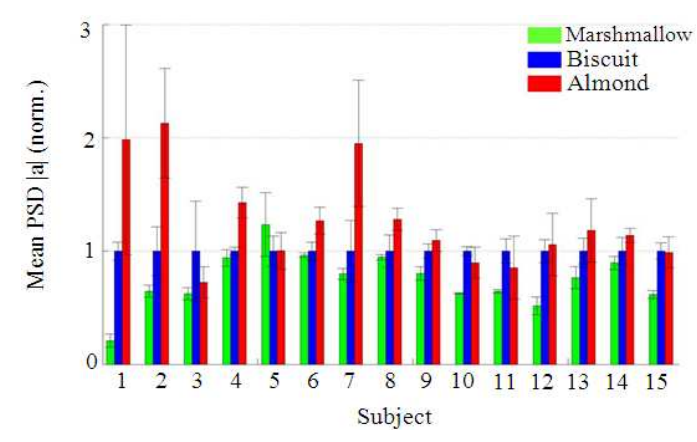

Fig. 11: Normalized Power Spectrum Density (PSD) of acceleration module $|\mathrm{a}|$ for 3 different foods, averaged on the 3 trials for each subject

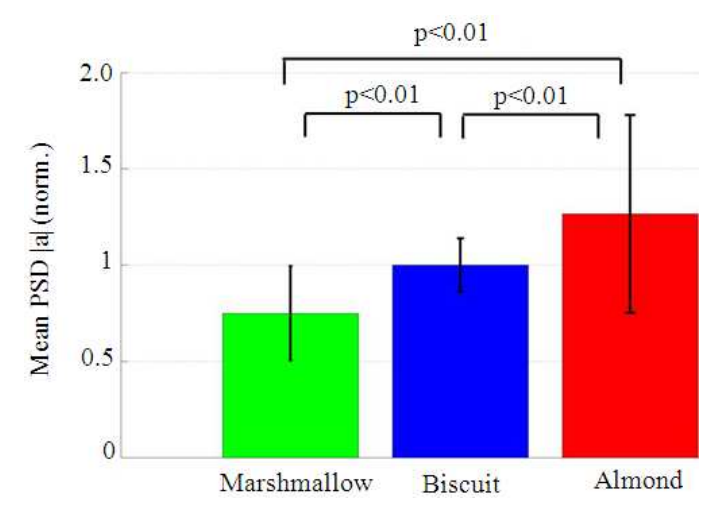

Fig. 12: Normalized Power Spectrum Density (PSD) of acceleration module $|\mathrm{a}|$ for 3 different foods, averaged on all subjects' trials for each food 


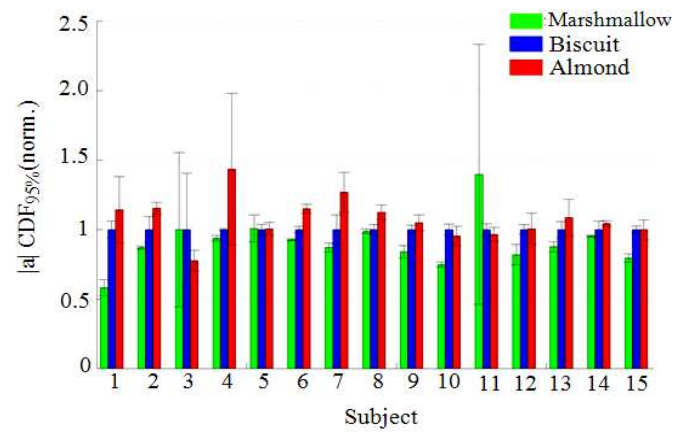

Fig. 13: Normalized 95\% Cumulative Distribution Function (CDF) of acceleration module $|\mathrm{a}|$ for 3 different foods, averaged on the 3 trials for each subject

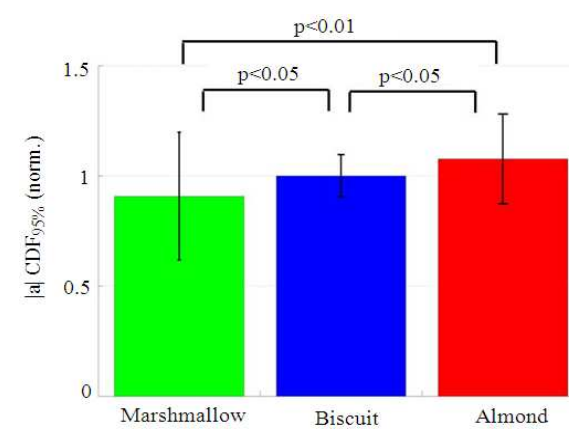

Fig. 14: Normalized 95\% Cumulative Distribution Function (CDF) of acceleration module $|\mathrm{a}|$ for 3 different foods, averaged on all subjects' trials for each food

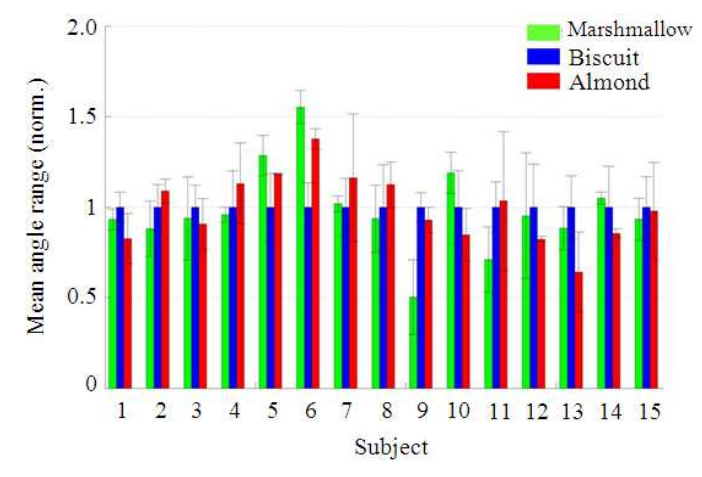

Fig. 15: Normalized mouth opening angle range for 3 different foods, averaged on the 3 trials for each subject

Figure 14 clearly shows that there was significant difference in $\mathrm{CDF}$ of acceleration while eating these three foods of different hardness. Subjects used high acceleration in chewing hard food.

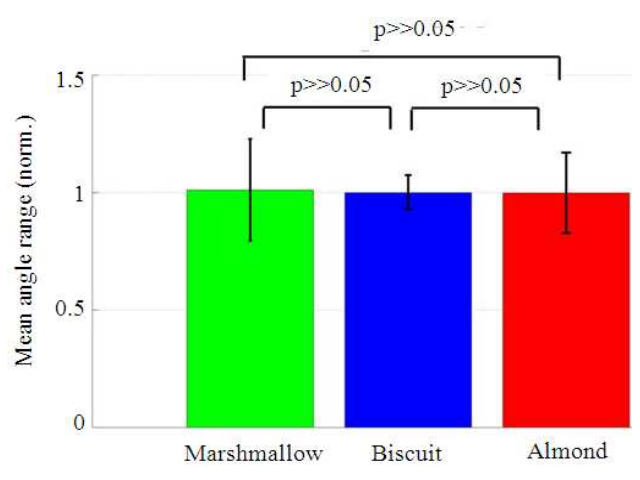

Fig. 16: Normalized mouth opening angle range for 3 different foods, averaged on all subjects' trials for each food

Mouth opening angle: The averaged mouth opening angle range was also evaluated, as shown in Fig. 15. There were no significant regular patterns of mouth opening angle in chewing these foods among all the subjects. Figure 16 further shows that there was no significant difference $(p>0.05)$ of the mouth opening angle range for eating these three foods. This may indicate that mouth opening angle is based on subject's fixed hyoid biomechanics, which leads to a regular eating habit in mouth opening that has less relation to the food of different hardness.

\section{DISCUSSION}

With the diffusion of more and more advanced tools and technologies in clinical diagnosis and treatment, it is fundamental to develop more efficient and handy devices or systems for routine tests. In this study, we presented the use of an ultra-miniaturized Inertial Measurement Unit WB-3 as a tool for jaw movement analysis. The extreme lightweight and its extreme high performance make WB-3 suitable for this application. Moreover, WB-3 directly acquires accelerations and rotations at high frequency, thus providing smooth and virtually noise-free data. Commercial systems, instead, acquire target's position and then obtain rotations and accelerations by successive derivations which introduce more noise.

As a preliminary test, we applied WB-3 to subject's mandible with minimal physical restriction to subject. We used this system to evaluate the jaw movements during free chewing of three types of food of different shapes and hardness (namely: Marshmallows, biscuits and almonds).

For this preliminary experiment we calculated and analyzed the following parameters, all related to the masticator efficiency and pattern while chewing 
different foods: Chewing Time (CT); Chewing Frequency (CF); Power Spectrum Density (PSD) of jaw's $\mathrm{x}$-axis angular speed $\omega_{\mathrm{x}}$ (sagittal plan) and acceleration module $|\mathrm{a}|$; Cumulative Distribution Function (CDF) of acceleration module $|\mathrm{a}|$ and Mouth Opening Angle (MOA).

The preliminary analysis of these parameters proved that several parameters extracted from the IMU's data allow a clear masticator efficiency and pattern analysis among these three foods of different hardness. In particular, the experimental results clearly show that the subjects used less chewing time, chewing frequency, acceleration CDF and energy to eat soft food; higher values were found in the case of hard food. However, there was no significant difference in mouth opening angle while eating these three foods.

It is important to notice that the subjects were instructed to lean his head on the wall during chewing the food in order to minimize the influence of head's movement. This constraint can be easily removed by using two IMUs, one on the mandible for the measurement and one on the head for movement compensation. In addition this setup would also allow the measurement of head movements during free mastication.

\section{CONCLUSION}

Our jaw movement analysis prototype using our ultra-miniaturized IMU WB-3 proved to be a valid and handy method for jaw movement and pattern analysis that may be used clinically as an assistant system for dental therapy. The experimental data could also show different jaw movement patterns among different subjects which may contribute to a further research on human's diet habits.

In the future, more experiments and analysis will be focused on chewing different shapes and weights of food. Moreover, the experiments on patients who had diseases in masticator systems are needed to further evaluate and verify the system validity

\section{ACKNOWLEDGMENT}

This research has been supported by the G-COE Global Robot Academia Program in Waseda University, Japan and by the Advancement of University Education Project of Chinese government Grant (2007)-3020. This research has been also partially supported by a Grant by STMicroelectronics, which also provided the core sensors and microcontroller. This research has been conducted at Humanoid Robotics Institute, in collaboration with the G-COE Global Robot Academia and S-COE ASMeW in Waseda University; partial support was also provided by the ASMeW Priority Research C Grant 11, by the JSPS Grant-in-aid for Scientific Research 19700389 and by Waseda University Grant for Special Research Projects (No. 266740).

The researchers would like to express their thanks to the Italian Ministry of Foreign Affairs, General Directorate for Cultural Promotion and Cooperation, for its support to RoboCasa. The authors would also like to express their gratitude to Okino Industries LTD, Japan ROBOTECH LTD, SolidWorks Corp, Dyden, for their support to the research.

Eventually, the authors would like to thank the 15 volunteers who kindly accepted to take part in the experiment.

\section{REFERENCES}

Flavel, S.C., M.A. Nordstrom and T.S. Miles, 2002. A simple and inexpensive system for monitoring jaw movements in ambulatory humans. J. Biomech., 35: 573-577. DOI: 10.1016/S0021-9290(01)00233-0

Ibanez, D.O., F.P. Baquerin, D.Y. Choi and C.N. Riviere, 2006. Performance envelope and physiological tremor in microsurgery. Proceeding of the IEEE 32nd Annual Northeast Bioengineering Conference, Apr. 1-2, IEEE Xplore Press, Easton, PA., USA, pp: 121-122. DOI: 10.1109/NEBC.2006.1629782

Kitamura, M., 2006. Study on jaw movement measurement in six-degree-of-freedom with intraoral sensor. Shikoku Dent. Res., 19: 67-75.

Kuska, B., 2005. NIDCR Launches Important Study on Temporomandibular Joint and Muscle Disorders. http://www.nidcr.nih.gov/Research/ResearchResult s/NewsReleases/ArchivedNewsReleases/NRY2005 /PR12052005

Marins, J.L., X. Yun, E.R. Bachmann, R.B. McGhee and M.J. Zyda, 2001. An extended Kalman filter for quanternion-based orientation estimation using MARG sensors. Proceeding of the IEEE/RSJ International Conference on Intelligent Robots and Systems, Oct. 29-Nov. 3, IEEE Xplore Press, Maui, Hawaii, USA., pp: 2003-2011. DOI: 10.1109/IROS.2001.976367.

Naeije, M. and N. Hofman, 2003. Biomechanics of the human temporomandibular joint during chewing. J. Dent. Res., 82: 528-531. DOI: $10.1177 / 1544059110308200708$.

Otake, Y., N. Suzuki, A. Hattori, Y. Shigeta and T. Ogawa et al., 2006. Real-time mandibular movement analysis system using four-dimensional cranial bone model. Syst. Comput. Jap., 37: 1-12. DOI: $10.1002 /$ scj.20582 
Sessa, S., M. Zecca, Z. Lin, T. Sasaki, K. Itoh and A. Takanishi, 2009. Waseda Bioinstrumentation system\#3 as a tool for objective rehabilitation measurement and assessment-development of the inertial measurement unit. Proceeding of the IEEE 11th International Conference on Rehabilitation Robotics, IEEE Xplore Press, Jun. 23-26, Kyoto, Japan, $\quad$ pp: $115-120$. DOI: 10.1109/ICORR.2009.5209491

Soboleva, U., L. Laurina and A. Slaidina, 2005. Jaw traking devices-historical review of methods development, Part I. Stomatologija, Baltic Dent. Maxillofacial J., 7: 67-71. PMID: 16340269

Veluvolu, K.C., U.X. Tan, W.T. Latt, C.Y. Shee and W.T. Ang, 2007. Bandlimited multiple fourier linear combiner for real-time tremor compensation. Proceeding of the IEEE 29th Annual International Conference Engineering in Medicine and Biology Society, Aug. 22-26, IEEE Xplore Press, Lyon, pp: 2847-2850.

10.1109/IEMBS.2007.4352922
Yabukami, S., H. Kanetaka, N. Tsuji, A. Itagaki and M. Yamaguchi et al., 2002. A new tracking system of jaw movement using two magnets. IEEE Trans. Magnet., 38: 3315-3317. DOI: 10.1109/TMAG.2002.802301

Zecca, M., S. Sessa, Z. Lin, T. Sasaki and T. Suzuki et al., 2009. Development of the ultra-miniaturized inertial measurement unit WB3 for objective skill analysis and assessment in neurosurgery: preliminary results. Lecture Notes Comput. Sci., 5761: 443-450. DOI: 10.1007/978-3-642-04268-3 